\title{
Estado nutricional e ingestão alimentar de pacientes em diálise peritoneal contínua com e sem hiperparatireoidismo secundário
}

\author{
Nutritional status and food intake of continuous peritoneal dialysis \\ patients with and without secondary hyperparathyroidism
}

\section{Autores \\ Sara Ribeiro Campos Maria Helena Lima Gusmão Alessandra Fortes Almeida Luís José Cardoso Pereira Lilian Ramos Sampaio Jairza Maria Barreto Medeiros}

Universidade Federal da Bahia - UFBA.

Data de submissão: 18/04/2011 Data de aprovação: 25/04/2012

\section{Correspondência para: Maria Helena Lima Gusmão Escola de Nutrição da Universidade Federal da Bahia Alameda Carrara, 23, apto. 1802 - Pituba Salvador - BA - Brasil CEP 41830-590 E-mail: mhlgusmao@ gmail.com}

Suporte financeiro: Universidade Federal da Bahia.

O referido estudo foi realizado no Complexo Hospitalar Universitário Professor Edgard Santos e na Clínica São Marcos Salvador - BA - Brasil.

Os autores declaram a inexistência de conflitos de interesse.

\section{Resumo}

Introdução: $O$ hiperparatireoidismo secundário (HPT2) é uma complicação comum e precoce em pacientes com doença renal crônica (DRC). Estudos têm sugerido que os altos níveis de paratormônio (PTH) podem exercer efeitos deletérios no estado nutricional de pacientes com DRC. Assim, o objetivo deste estudo foi comparar o estado nutricional de pacientes com DRC em diálise peritoneal contínua (DPC) com e sem HPT2. Objetivo: O objetivo deste estudo é comparar o estado nutricional de pacientes com DRC em diálise peritoneal contínua (DPC) com e sem HPTS. Métodos: Foram avaliados 74 pacientes, entre adultos e idosos, divididos em dois grupos, de acordo com os níveis de PTH. No grupo 1 (n =18) os níveis de PTH eram maiores do que $300 \mathrm{pg} / \mathrm{mL}$ e, no grupo 2, menores ou iguais a $300 \mathrm{pg} / \mathrm{mL}$. O estado nutricional dos pacientes foi avaliado através da antropometria, exames bioquímicos e avaliação subjetiva global (ASG). O consumo alimentar foi avaliado através do registro alimentar de 3 dias. Foram coletados também dados clínicos, como cálcio e fósforo séricos, e a presença de inflamação foi avaliada através da dosagem de proteína $\mathrm{C}$ reativa ultrassensível (PCR US). Resultados: A média de idade da população estudada foi de $54,97 \pm 17,06$ anos, com predominância de pacientes adultos $(58,1 \%)$ e do sexo feminino $(56,8 \%)$. O tempo de DPC expresso em mediana foi de 17 meses (7,75-33). Através da ASG, demonstrou-se prevalência de $36,5 \%$ de desnutridos na população. $\mathrm{Na}$ análise de diferença entre os grupos, em relação à antropometria, indicadores bioquímicos e ASG, bem como ingestão alimentar, não foram evidenciadas diferenças entre os grupos estudados. Conclusão: Não houve diferenças no estado nutricional e na ingestão alimentar entre os pacientes com e sem HPT2.

Palavras-chave: Estado nutricional. Ingestão de alimentos. Diálise peritoneal. Hiperparatireoidismo secundário.

\section{Abstract}

Introduction: Secondary hyperparathyroidism (SHPT) is a common and early complication in chronic kidney disease (CKD) patients. Studies have suggested that high levels of parathyroid hormone (PTH) may have deleterious effects on the nutritional status of patients with CKD. Thus, the aim of this study was to compare the nutritional status of CKD patients in continuous peritoneal dialysis (CPD) with and without SHPT. Objective: The aim of this study was to compare the nutritional status of CKD patients in continuous peritoneal dialysis (CPD) with and without SHPT. Methods: 74 patients were evaluated, including adults and seniors, divided into two groups according to PTH levels. In group $1(n=18)$ levels of PTH higher than $300 \mathrm{pg} / \mathrm{mL}$ and in group 2 less than or equal to $300 \mathrm{pg} / \mathrm{mL}$. The nutritional status of patients was assessed by anthropometry, biochemical and subjective global assessment (SGA). Food intake was measured by food record for 3 days. We also collected clinical data, such as calcium and serum phosphorus, and the presence of inflammation was assessed by measuring C-reactive protein ultra-sensitive (CRP US). Results: The average age of the population studied was 54.97 \pm 17.06 years, with predominance of adult patients $(58.1 \%)$ and females $(56.8 \%)$. The time of CPD expressed in median was 17 months (7.75-33). Through ASG, it was shown a prevalence of $36.5 \%$ of the population undernourished. In the analysis of difference between the groups in relation to anthropometry, biochemical indicators and ASG as well as food intake, there were no differences between the groups. Conclusion: There were no differences in nutritional status and dietary intake between patients with and without SHPT.

Keywords: Nutritional status. Eating. Peritoneal dialysis. Hyperparathyroidism, secondary. 


\section{INTRODUÇ̃̃O}

O hiperparatireoidismo secundário (HPT2) é uma complicação comum e precoce em pacientes portadores de doença renal crônica (DRC), a qual implica em alta morbimortalidade e dificuldade de controle médico. ${ }^{1}$ O HPT2 caracteriza-se pela hiperplasia das glândulas paratireoides e hipersecreção de paratormônio $(\mathrm{PTH})$. Dentre os fatores associados à patogênese do HPT2, destacam-se a deficiência de vitamina D, hiperfosfatemia, hipocalcemia, alterações dos receptores de cálcio $(\mathrm{CaR})$ e de vitamina $\mathrm{D}(\mathrm{VDR})$ das paratireoides, resistência à ação do PTH e níveis elevados do hormônio fibroblast growth factor 23 (FGF-23).,2,3

As alterações fisiopatológicas causadas pela doença resultam em quadro clínico característico, que inclui principalmente dores ósseas e musculares, prurido, calcificação vascular, fraturas ósseas, redução do apetite, alterações do paladar e perda de peso. ${ }^{4,5}$

Além disso, alterações comumente observadas em pacientes dialíticos, como ingestão alimentar deficiente, perdas de nutrientes durante a diálise, estado inflamatório crônico, intolerância à glicose e distúrbios hormonais, podem ser fatores agravantes e contribuintes para o desenvolvimento da desnutrição energético-proteica (DEP) em indivíduos com HPT2. ${ }^{6}$

Peters et al. ${ }^{7}$, ao estudarem os efeitos do HPT2 na composição corporal de pacientes portadores de DRC em programa de hemodiálise, concluíram que os altos níveis de PTH contribuem para os efeitos deletérios na composição corporal de pacientes renais crônicos e piora do seu estado nutricional.

A DEP é uma complicação frequente em pacientes mantidos em diálise peritoneal contínua (DPC). A prevalência de desnutrição varia de 32,9 a 51,2\% na forma leve a moderada e de 2,6 a $4,2 \%$ para desnutrição grave. ${ }^{8-10}$

Alterações metabólicas e nutricionais decorrentes da doença e da própria terapia dialítica, como perda de proteínas, aminoácidos e vitaminas hidrossolúveis, supressão do apetite em virtude da absorção contínua de glicose do dialisato, desconforto abdominal, peritonites, diálise pouco eficiente, sobrecarga hídrica, inflamação, acidose metabólica e o HPT2 estão entre as causas da DEP na DPC. ${ }^{11,12}$

Alguns estudos experimentais em animais têm demonstrado os efeitos deletérios do PTH no metabolismo proteico $;^{13,14}$ porém, pouco tem sido estudado acerca dos efeitos do HPT2 no estado nutricional de pacientes em diálise.

Considerando assim, que a DEP em pacientes mantidos em DPC constitui um fator de risco para sobrevida e qualidade de vida desses indivíduos e possui associação com o maior risco de morte, ${ }^{9,14}$ bem como a escassez de estudos sobre o estado nutricional de pacientes renais crônicos em diálise peritoneal com HPT2, este estudo teve como objetivo comparar o estado nutricional de pacientes com DRC em diálise peritoneal contínua com e sem HPT2.

\section{Pacientes e Métodos}

Tratou-se um estudo transversal observacional, realizado nas unidades de tratamento dialítico do Hospital Universitário Professor Edgard Santos (HUPES) e da Clínica São Marcos (CSM), Salvador (BA), no período de março a agosto de 2007, envolvendo 74 pacientes.

Participaram do estudo pacientes adultos $(\geq 20 \mathrm{e}$ $<60$ anos) e idosos ( $\geq 60$ anos) de ambos os sexos, em diálise peritoneal ambulatorial contínua (CAPD) ou diálise peritoneal automatizada (DPA) há pelo menos 3 meses, em uso de dieta via oral e com clearance de creatinina menor do que $10 \mathrm{~mL} / \mathrm{min}$ para pacientes não diabéticos e menor do que $15 \mathrm{~mL} / \mathrm{min}$ para pacientes diabéticos.

Foram considerados critérios de exclusão, possuir doença maligna, síndrome da imunodeficiência adquirida e/ou doença inflamatória crônica, abandono da terapia dialítica nos últimos três meses, alcoolismo, insucesso no transplante renal nos últimos seis meses, uso de corticoide, uso de nutrição enteral e/ou nutrição parenteral exclusiva ou associada à dieta via oral.

Para caracterizar a população, foram utilizados dados demográficos, como idade e sexo, e dados clínicos, como a etiologia da doença renal, o tipo de DPC, o tempo de DPC em meses. Foram analisados também dados sobre a adequação da terapia dialítica, através do índice de remoção da ureia (KT/V total) e do clearance de creatinina total semanal; assim como alterações do metabolismo mineral e ósseo, através da dosagem de PTH, cálcio e fósforo séricos e do produto cálcio e fósforo. Os níveis séricos de proteína $\mathrm{C}$ reativa ultrassensível (PCR US) foram usados como marcadores de inflamação nos pacientes incluídos no estudo.

A avaliação da terapia dialítica e dos níveis séricos de PTH, cálcio, fósforo e produto cálcio e fósforo seguiram a técnica e recomendação da National Kidney Foundation's Kidney Disease Outcomes Quality Initiative (KDOQI). ${ }^{15,16}$

Para análise do PTH intacto, foi utilizado o método da quimioluminescência e para as dosagens de cálcio e fósforo, o método titulométrico e de Basques e Lustosa, respectivamente. Os níveis de cálcio foram 
corrigidos pelos níveis de albumina sérica. ${ }^{16} \mathrm{~A}$ dosagem de albumina foi realizada pelo método verde de bromocresmol. Os valores foram comparados com os referenciados pelo KDOQI. ${ }^{16}$

A avaliação da presença de inflamação seguiu os valores de PCR US, pelo método nefelometria, conforme referência da IV Diretrizes Brasileiras sobre Dislipidemia e Diretriz de Prevenção da Aterosclerose da Sociedade Brasileira de Cardiologia. ${ }^{17}$

Os pacientes foram submetidos a uma avaliação nutricional, realizada por equipe treinada, que constou de avaliação antropométrica, parâmetros bioquímicos, avaliação subjetiva global (ASG) e consumo alimentar.

\section{AvALIAÇÃO ANTROPOMÉTRICA}

Para avaliação antropométrica da população foram utilizadas a circunferência do braço $(\mathrm{CB})$, prega cutânea tricipital (PCT), circunferência muscular do braço $(\mathrm{CMB})$, área muscular do braço corrigida $(\mathrm{AMBc}) \mathrm{e}$ área de gordura do braço (AGB).

A CB foi aferida com uma fita de fibra de vidro com $5 \mathrm{~cm}$ de largura no ponto médio entre o acrômio e o olecrano, segundo as recomendações e referências da Organização Mundial de Saúde (OMS). ${ }^{18}$ Os valores obtidos da CB favoreceram os cálculos da AMBc e $\mathrm{CMB}$ segundo referências de Frisancho. ${ }^{19}$ Os padrões de normalidade utilizados para $\mathrm{CMB}$ seguiram as referências do Third National Health and Nutrition Examination Survey (NHANES III). ${ }^{20}$

A PCT foi aferida utilizando o adipômetro Lange calibrado antes e após a coleta diária de dados utilizando-se um bloco calibrador, segundo as recomendações e referências da OMS. ${ }^{18}$

Os valores obtidos das pregas cutâneas favoreceram o cálculo da AGB para adultos segundo referências de Frisancho ${ }^{19}$ e a estimativa de excesso de tecido adiposo em idosos, de acordo com os percentis do NHANES III. ${ }^{20} \mathrm{O}$ valor obtido da PCT também favoreceu o cálculo da AMBc e da CMB.

\section{ParÂMetros bioouímicos}

Os valores das dosagens séricas de ureia, creatinina e albumina foram coletados dos prontuários médicos dos pacientes, após a sua consulta mensal. Os valores de ureia, creatinina e albumina seguiram as recomendações propostas pelo K/DOQI. ${ }^{21}$

\section{Avaliação subJetiva global}

Foi realizada por meio de modelo proposto pelo Estudo CANUSA (CANADA-USA: peritoneal dialysis study group), ${ }^{8}$ descrito como uma escala de 4 itens e 7 pontos, que permite a classificação em desnutrição grave, leve ou moderada e estado nutricional adequado. ${ }^{8,21} \mathrm{O}$ Guidelines da National Kidney Foundation ${ }^{21}$ recomenda o uso desse instrumento devido a sua melhor sensibilidade.

\section{INQUÉRITO ALIMENTAR}

A avaliação da ingestão alimentar habitual foi realizada utilizando-se o registro alimentar de três dias (dois dias durante a semana e 1 dia do final de semana). Para o cálculo da ingestão de nutrientes, foi utilizado o Programa Diet Pro versão 4.0.22 Nesse programa utilizou-se como referência a Tabela Americana de Composição dos Alimentos. Alimentos regionais ausentes na tabela foram acrescidos ao programa, utilizando-se a Tabela de Composição Química dos Alimentos do Estudo Nacional da Despesa Familiar ${ }^{23}$ ou a Tabela de Composição Química dos Alimentos de Philippi. ${ }^{24}$ A glicose absorvida do dialisato também foi acrescida ao consumo alimentar. Essa glicose foi estimada pela equação proposta por Grodstein et al. ${ }^{25}$ As médias de consumo de calorias, proteínas, carboidratos, lipídeos, cálcio e fósforo foram calculadas através do Programa Diet Pro versão 4.0 e comparadas às recomendações específicas para pacientes renais segundo Martins. ${ }^{26}$ Já, os valores de absorção de glicose foram comparados às estimativas de Dinamod e Henrich. ${ }^{27}$ Como parâmetro de referência para o consumo alimentar de carboidrato, foi utilizada a recomendação específica para pacientes renais em DPC. ${ }^{12}$

Para identificar a presença de HTP2, foi realizada a dosagem de PTH intacto (PTHi), utilizando-se o método da quimioluminescência. Foram considerados com HTP2 aqueles pacientes com nível sérico de PTHi superior a $300 \mathrm{pg} / \mathrm{mL}$, pois o $\mathrm{KDOQI}^{16}$ reco- $^{-}$ menda que os indivíduos com DRC estágio 5 mantenham os níveis de PTH sérico entre 150 - 300 pg/mL.

\section{AnÁlise estatística}

Os dados foram apresentados como média \pm desvio-padrão ou mediana para variáveis contínuas e percentagem para variáveis categóricas. Para análise estatística, os dados obtidos foram classificados, por meio do teste de Kolmogorov-Smirnov, como de distribuição normal (dados paramétricos) e não normal (dados não paramétricos). Na comparação entre dois grupos foi utilizado o teste $t$ de Student para os dados paramétricos e de Mann-Whitney para os não paramétricos. As proporções foram comparadas pelo teste do qui-quadrado ou teste 
exato de Fisher. O Programa Statistical Package for Social Science (SPSS ${ }^{\circledR}$, versão 13.0) foi utilizado para todas as análises estatísticas, utilizando-se uma significância de $5 \%(\mathrm{p}<0,05)$.

O protocolo deste estudo foi aprovado pelo Comitê de Ética em Pesquisa da Escola de Nutrição da Universidade Federal da Bahia.

\section{Resultados}

A média de idade da população estudada foi de 54,97 $\pm 17,06$ anos, com predominância de pacientes adultos $(58,1 \%)$ e do sexo feminino $(56,8 \%)$. Quanto à etiologia da DRC, observou-se que $36,5 \%$ dos pacientes tiveram como causa da DRC a nefropatia hipertensiva, $24,3 \%$ a nefropatia diabética, $23 \%$ as doenças primárias (glomerulopatias) e 8,2\% referiram outras causas. Em relação à modalidade de DPC, 83,8\% realizavam CAPD, enquanto que $16,2 \%$ faziam DPA. O tempo de DPC expresso em mediana foi de 17 meses $(7,75-33)$.

Dentre os pacientes avaliados, apenas $22,7 \%$ possuíam informação sobre adequação dialítica no prontuário. A análise de comparação entre o grupo que apresentava registro de adequação e o que não apresentava demonstrou diferença significativa somente em relação ao tempo de diálise $(\mathrm{p}=0,010)$, sendo de 30 meses $(20,5$ - 49,5) para o grupo com adequação e de 12 meses $(6-27,5)$ para o grupo sem registro de adequação. O KT/V médio da população foi de 2,40 土 0,56 e o clearance de creatinina semanal médio foi de $61,60 \pm 20,09 \mathrm{~L} / 1,73 \mathrm{~m}^{2}$.

O nível sérico de PTHi na população expresso em mediana foi de $168,5 \mathrm{pg} / \mathrm{mL}(76,5$ - 286,5) e esse esteve elevado em $24,3 \%$ dos pacientes. O cálcio e o fósforo séricos e o produto cálcio e fósforo, expressos em médias foram de 9,1 $\pm 1,09 \mathrm{mg} / \mathrm{dL}, 4,41 \pm$ $1,2 \mathrm{mg} / \mathrm{dL}$ e $40,29 \pm 12,94 \mathrm{mg}^{2} / \mathrm{dL}^{2}$, respectivamente, e $54,1 \%$ dos pacientes apresentaram níveis de PCR US elevados (> $3 \mathrm{mg} / \mathrm{L}),{ }^{17}$ sendo seu valor expresso em mediana de 3,95 mg/dL $(1,03-9,5)$.
Em relação ao estado nutricional, a avaliação antropométrica dos indivíduos adultos permitiu verificar que 9,3\% desses apresentavam déficit de tecido adiposo (AGB < P5) e 34,9\% apresentavam depleção de tecido muscular $(\mathrm{AMBc}<\mathrm{P} 5)$. Dentre os indivíduos idosos, observou-se que o déficit de tecido adiposo (PCT < P10) esteve presente em 22,6\% dos mesmos e a depleção de tecido muscular $(\mathrm{CMB}<$ P10) em 38,7\%.

Os parâmetros bioquímicos demonstraram que o nível sérico de albumina foi em média 3,01 \pm $0,54 \mathrm{mg} / \mathrm{dL}$, de forma que a hipoalbuminemia esteve presente em $78,4 \%$ da população. Os valores médios de ureia e creatinina foram de $110,51 \pm 29,65 \mathrm{mg} / \mathrm{dL}$ e 9,37 $\pm 3,7 \mathrm{mg} / \mathrm{dL}$, respectivamente.

A avaliação nutricional da população, realizada através da ASG, demonstrou a frequência de 36,5\% de DEP, sendo predominantemente na forma leve a moderada $(35,1 \%)$.

Quanto ao perfil de ingestão alimentar, observou-se que a população estudada apresentou ingestão calórica média de 1799,2 $\pm 618,76 \mathrm{kcal} / \mathrm{dia}$. De acordo com a distribuição percentual dos macronutrientes, identificou-se que $64,32 \pm 8,11 \%$ correspondiam aos carboidratos, destes $45,95 \pm 9,43 \%$ foram provenientes da dieta e 18,35 $\pm 9,72 \%$ oriundos da glicose absorvida do dialisato. O consumo de proteínas foi de $13,58 \pm 3,63 \%$ e o de lipídio representou 22,25 $\pm 6,83 \%$ do valor calórico total (VCT). O consumo médio diário de cálcio foi de $579,18 \pm 328,54 \mathrm{mg}$ e o de fósforo foi de 773,05 $\pm 369,2 \mathrm{mg}$.

Quando se dividiu a população em dois grupos, Grupo 1 - PTHi acima de 300 pg/mL ( $\mathrm{n}=18$ ) e Grupo 2 - PTHi abaixo de 300 pg/mL ( $\mathrm{n}=56$ ), observou-se que o cálcio e o fósforo séricos, o produto cálcio e fósforo e os níveis séricos de PCR US não apresentaram diferença entre os grupos, conforme demonstrado na Tabela 1.

Os índices de déficit de tecido adiposo e de massa muscular em adultos e idosos, conforme as Tabelas 2

Tabela 1 PARÂMETROS CLÍNICOS DE PACIENTES ADULTOS E IDOSOS EM DIÁLISE PERITONEAL COM E SEM HIPERPARATIREOIDISMO SECUNDÁRIO. SALVADOR, $2007(n=74)$

\begin{tabular}{|c|c|c|c|}
\hline & $\begin{array}{l}\text { Grupo } 1 \\
(n=18)\end{array}$ & $\begin{array}{c}\text { Grupo } 2 \\
(n=56)\end{array}$ & Valor $\mathrm{p}$ \\
\hline & Média \pm DP ou mediana & Média \pm DP ou mediana & \\
\hline Cálcio sérico (mg/dL) & $9,16 \pm 0,80$ & $9,08 \pm 1,18$ & 0,77 \\
\hline Fósforo sérico (mg/dL) & $4,76 \pm 1,63$ & $4,30 \pm 1,01$ & 0,28 \\
\hline $\mathrm{Ca} \times \mathrm{P}\left(\mathrm{m}^{2} / \mathrm{dL}^{2}\right)$ & $43,53 \pm 15,86$ & $39,25 \pm 11,83$ & 0,22 \\
\hline PCR US (mg/L) & $5,89(1,97-10,55)$ & $2,76(0,61-8,77)$ & 0,07 \\
\hline
\end{tabular}

DP: desvio padrão; Ca x P: produto cálcio e fósforo; PCR US: proteína $C$ reativa ultrassensível. 
e 3, respectivamente, também não apresentaram diferenças entre os grupos.

$\mathrm{Na}$ análise de diferença entre os grupos, em relação aos indicadores bioquímicos e ASG, bem como ingestão alimentar dos pacientes, conforme as Tabelas $4 \mathrm{e}$ 5 , respectivamente, também não foram evidenciadas diferenças entre os grupos estudados.

\section{Tabela 2 PARÂMETROS ANTROPOMÉTRICOS DE PACIENTES ADULTOS EM DIÁLISE PERITONEAL COM E SEM HIPERPARATIREOIDISMO SECUNDÁRIO. SALVADOR, $2007(n=43)$}

\begin{tabular}{ll}
$\begin{array}{ll}\text { Grupo } 1 \\
(\mathrm{n}=11)\end{array}$ & $\begin{array}{l}\text { Grupo } 2 \\
(\mathrm{n}=32)\end{array}$ \\
\cline { 1 - 3 }$\%(\mathrm{n})$ & $\%(\mathrm{n})$
\end{tabular}

$\mathrm{AMBc}$

Déficit $(<P 5) \quad 27,3(3) \quad 37,5(12) \quad 0,71$
AGB

Déficit (<P5) 9,1 (1)

$9,4(3) \quad 1,00$

AMBc: área muscular do braço corrigida; $A G B$ : área de gordura do braço.

\section{Discussão}

O HPT2 é uma grande complicação clínica em pacientes com DRC, primeiramente devido a sua prevalência, uma vez que quase todos os indivíduos renais crônicos apresentam algum grau desse distúrbio..$^{28}$ No Brasil, a prevalência do HPT2 é crescente.

\section{Tabela 3 PARÂMETROS ANTROPOMÉTRICOS DE PACIENTES IDOSOS EM DIÁLISE PERITONEAL COM E SEM HIPERPARATIREOIDISMO SECUNDÁRIO. SALVADOR, $2007(\mathrm{n}=31)$}

\begin{tabular}{lccc} 
& $\begin{array}{c}\text { Grupo } 1 \\
(\mathrm{n}=7)\end{array}$ & $\begin{array}{c}\text { Grupo } 2 \\
(\mathrm{n}=24)\end{array}$ & Valor p \\
\cline { 2 - 3 } & $\%(\mathrm{n})$ & $\%(\mathrm{n})$ & \\
\hline CMB & & & \\
$\quad \begin{array}{c}\text { Déficit (<P10) } \\
\text { PCT }\end{array}$ & $28,6(2)$ & $41,7(10)$ & 0,67 \\
$\quad$ Déficit (<P10) & $42,9(3)$ & $16,7(4)$ & 0,30 \\
\hline
\end{tabular}

CMB: circunferência muscular do braço; PCT: prega cutânea tricipital.

\begin{tabular}{|c|c|c|c|c|}
\hline & \multicolumn{4}{|c|}{$\begin{array}{l}\text { PARÂMETROS BIOQUímICOS E AVALIAÇÃo SUBJETIVA GLOBAL DE PACIENTES ADULTOS E IDOSOS EM DIÁLISE } \\
\text { PERITONEAL COM E SEM HIPERPARATIREOIDISMO SECUNDÁRIO. SALVADOR, } 2007(n=74)\end{array}$} \\
\hline & & Grupo $1(n=18)$ & Grupo $2(n=56)$ & \multirow{2}{*}{ Valor $\mathrm{p}$} \\
\hline & & Média \pm DP ou $\%(n)$ & Média \pm DP ou $\%(n)$ & \\
\hline \multicolumn{5}{|c|}{ Bioquímicos } \\
\hline \multicolumn{2}{|c|}{ Albumina sérica (g/dL) } & $3,00 \pm 0,61$ & $3,02 \pm 0,53$ & 0,88 \\
\hline \multicolumn{2}{|c|}{ Ureia sérica (mg/dL) } & $121,76 \pm 38,56$ & $108,16 \pm 25,01$ & 0,09 \\
\hline \multicolumn{2}{|c|}{ Creatinina sérica $(\mathrm{mg} / \mathrm{dL})$} & $10,78 \pm 3,85$ & $8,85 \pm 3,53$ & 0,57 \\
\hline \multicolumn{5}{|c|}{ ASG } \\
\hline \multicolumn{2}{|c|}{ Desnutridos } & $33,3(6)$ & $37,5(21)$ & 1,00 \\
\hline
\end{tabular}

DP: desvio padrão; ASG: avaliação subjetiva global.

Tabela 5 PADRÃO dE CONSUMO ALIMENTAR DE PACIENTES ADULTOS E IDOSOS EM DIÁLISE PERITONEAL COM E SEM HIPERPARATIREOIDISMO SECUNDÁRIO. SALVADOR, $2007(n=74)$

\begin{tabular}{lccc} 
& Grupo $1(\mathrm{n}=18)$ & Grupo $2(\mathrm{n}=56)$ & Valor $\mathrm{p}$ \\
\cline { 2 - 3 } & Média $\pm \mathrm{DP}$ & Média \pm DP & 0,64 \\
\cline { 2 - 3 } VCT (kcal/dia) & $1857,63 \pm 557,82$ & $1780,43 \pm 640,71$ & 0,92 \\
Carboidrato alimentar (\% VCT) & $45,76 \pm 10,97$ & $46,01 \pm 8,99$ & 0,95 \\
Glicose absorvida do dialisato (\% VCT) & $18,24 \pm 10,31$ & $18,38 \pm 9,61$ & 0,84 \\
Carboidratos totais (\% VCT) & $64 \pm 8,32$ & $64,43 \pm 8,11$ & 0,35 \\
Proteínas (\% VCT) & $12,89 \pm 2,84$ & $13,80 \pm 3,85$ & 0,56 \\
Lipídios (\% VCT) & $23,07 \pm 7,53$ & $21,99 \pm 6,65$ & 0,31 \\
Cálcio (mg/dia) & $510,77 \pm 316,11$ & $601,17 \pm 332,20$ & 0,97 \\
Fósforo (mg/dia) & $775,42 \pm 322,66$ & $772,29 \pm 385,67$ & \\
\hline
\end{tabular}

DP: desvio padrão; VCT: valor calórico total. 
Araújo et al. ${ }^{29}$ ao estudarem padrões histológicos da osteodistrofia renal, mostraram que as lesões ósseas devido ao HPT2 aumentaram de $32,3 \%$ na década de 1980 para $44 \%$ nos anos 1990 .

No presente estudo, $24,3 \%$ dos pacientes apresentaram níveis elevados de PTHi. Os dados disponíveis sobre a prevalência do HPT2 são bastante variáveis. Sherrard et al. ${ }^{30}$ e Delmez et al. ${ }^{31}$ demonstraram que aproximadamente um terço dos pacientes em CAPD desenvolvem essa complicação; porém, em estudo mais recente realizado no Canadá, verificou-se uma prevalência de $47 \%$ de $\mathrm{HPT}^{32}$. De acordo com Malberti et al., ${ }^{33}$ a incidência do HPT2 aumenta com o tempo de diálise, portanto, a menor prevalência de HPT2 pode ter sido atribuída, em parte, ao menor tempo de diálise da nossa população do que foi reportado em outros trabalhos.

O PTH pode exercer um efeito direto no músculo esquelético, uma vez que a administração desse hormônio tem demonstrado afetar a produção, transferência e utilização de energia e o metabolismo de proteínas e aminoácidos no músculo esquelético de ratos. ${ }^{13,14}$ Portanto, os níveis persistentemente altos de PTH podem contribuir diretamente para a desnutrição, já que promovem alteração no metabolismo proteico e energético, conduzindo à perda de peso, fraqueza e atrofia muscular. ${ }^{13}$

O PTH é também conhecido por induzir a produção de interleucinas no fígado de ratos e aumentar os níveis circulantes dessas citocinas in vivo. Elevados níveis de interleucina-6 em indivíduos idosos têm sido recentemente associados com menor massa muscular e reduzida força muscular. ${ }^{34}$

Os estudos de Ritz et al., ${ }^{35}$ Visser et al. ${ }^{34}$ e Rezende et al. ${ }^{36}$ demonstraram que altos níveis de PTH podem, direta ou indiretamente, exercer uma ação tóxica no metabolismo energético e proteico, induzindo o catabolismo e o balanço nitrogenado negativo, bem como alterações na composição corporal, aumentando, por conseguinte, as limitações funcionais e a mortalidade.

O presente estudo identificou que o déficit nutricional na população estudada ainda é frequente. Os parâmetros antropométricos evidenciaram prevalência considerável de déficit de massa muscular e tecido adiposo entre adultos e idosos, porém, não foram encontrados dados sobre o estado nutricional da população em DP através da antropometria para fins de comparação, já que os estudos utilizam a ASG como indicador nutricional.

A hipoalbuminemia foi bastante prevalente entre os pacientes $(78,4 \%)$, porém, o nível de albumina da população geral apresentou valor médio superior ao achados de Wang et al. ${ }^{10} \mathrm{~A}$ creatinina sérica apresentou valor médio abaixo do recomendado pelo $\mathrm{KDOQI}^{21}$ ( $\geq 10 \mathrm{mg} / \mathrm{dL}$ ). Níveis séricos reduzidos de creatinina sugerem ingestão proteica insuficiente e/ou diminuição da massa muscular e está associada com aumento das taxas de mortalidade em pacientes renais crônicos. ${ }^{21}$

Por meio da ASG verificou-se uma frequência importante de desnutrição entre a população estudada, mas, ainda assim, o percentual encontrado foi menor do que o observado em alguns estudos, onde a desnutrição alcançou índices de 44,2 e 73,6\%, utilizando-se esse mesmo instrumento de avaliação. ${ }^{10,37} \mathrm{~A}$ discordância entre o resultado encontrado e o relatado pela literatura pode ter sido influenciado pelas diferentes características clínicas da população.

Existem poucos dados disponíveis sobre o padrão de consumo alimentar de pacientes em DPC. Em nosso estudo, o ponto mais relevante em relação à ingestão alimentar foi a alta contribuição dos carboidratos para o VCT. Observamos que o consumo alimentar desse macronutriente foi superior a $35 \%^{12}$ e a quantidade de glicose absorvida encontrou-se dentro do percentual estimado, de $20-30 \%$ do VCT. ${ }^{27}$ Observouse também consumo de cálcio e fósforo abaixo do recomendado para pacientes em diálise. ${ }^{16}$

Quando se dividiu a amostra em dois grupos, não foi observado diferenças no estado nutricional e na ingestão alimentar.

Rezende et al. ${ }^{36}$, ao estudarem o efeito do HPT2 no estado nutricional de pacientes com HPT2, observaram que quanto maior o nível de PTH menor a gordura corporal dos indivíduos em hemodiálise. Vale ressaltar que, até então, não existem estudos que avaliem os efeitos do HPT2 no estado nutricional da população em DPC.

A associação entre a inflamação crônica e os distúrbios do metabolismo mineral e ósseo tem sido documentada. ${ }^{38}$ Sabe-se, também, que pacientes desnutridos frequentemente possuem sinais de inflamação, caracterizada por aumento nos níveis plasmáticos de PCR e um desequilíbrio entre citocinas pró- e anti-inflamatórias, ${ }^{39}$ porém, não foram observadas diferenças entre os níveis de PCR US entre os grupos. Isso pode ter contribuído para as semelhanças nos itens da avaliação nutricional dos pacientes com e sem HPT2.

Algumas peculiaridades presentes nos pacientes em DP podem contribuir para a maior preservação do seu estado nutricional. A glicose do dialisato é absorvida em larga escala, já que uma diálise peritoneal convencional resulta em uma situação metabólica quase única, envolvendo absorção de glicose durante $24 \mathrm{~h}$ contínuas. ${ }^{40}$ Sendo assim, esses pacientes apresentam ao longo do tempo alterações na composição corporal, 
com aumento do peso corporal às custas de tecido adiposo e redução do nitrogênio corporal total, acarretando na perda de massa magra e proteínas. ${ }^{11,12}$

Os dados sobre a evolução das doenças ósseas em hemodiálise têm sido bem documentados, porém, a história natural do HPT2 na DPC ainda é incerta. ${ }^{41}$ Sendo assim, os resultados encontrados podem ser indicativos de que o HPT2 na DPC apresenta aspectos clínicos divergentes do que tem sido demonstrado em pacientes mantidos em hemodiálise.

\section{Conclusão}

No presente estudo, não houve diferenças no estado nutricional e na ingestão alimentar entre os pacientes em diálise peritoneal contínua com e sem HPT2. Outros estudos que abordem a relação entre estado nutricional e HPT2 considerando o pareamento da amostra por sexo, idade, tempo de diálise, bem como o controle das variáveis que interferem no estado nutricional, são ainda necessários.

\section{ReferênCIAS}

1. Sarkis KS, Aniteli TM, Peters BSE, Jorgetti V, Martini LA. Nutritional evaluation in secondary hyperparathyroidism patients. Rev Bras Nutr Clin 2007;22:197-202.

2. Martin KJ, González EA. Metabolic bone disease in chronic kidney disease. J Am Soc Nephrol 2007; 18:875-85.

3. Silver J, Kilav R, Naveh-Many T. Mechanisms of secondary hyperparathyroidism. Am J Physiol Renal Physiol 2002;283:F367-76.

4. Llach F, Massry SG. On the mechanism of secondary hyperparathyroidism in moderate renal insufficiency. J Clin Endocrinol Metabol 1985;61:601-6.

5. Coladonato JA, Ritz E. Secondary hyperparathyroidism and its therapy as a cardiovascular risk factor among end-stage renal disease patients. Adv Ren Replace Ther 2002;9:193-9.

6. Avesani CM, Heimburguer O, Stenvinkel P, Lindholm, B. Nutritional aspects of adult patients treated with chronic peritoneal dialysis. J Bras Nefrol 2007;18:232-8.

7. Peters BSE, Jorgetti V, Martini LA. Influência do hiperparatireoidismo secundário grave no estado nutricional de pacientes com insuficiência renal crônica. Rev Nutr 2006;19:111-8.

8 Canada-USA (CANUSA): Peritoneal dialysis study group: Adequacy of dialysis and nutition in continuous peritoneal dialysis: Association with clinical outcomes. J Am Soc Nephrol 1996;7:189-207.

9. Chan JYW, Che KI, Lam KMC, et al. Comprehensive malnutrition inflammation score as a marker of nutritional status in Chinese peritoneal dialysis patientes. Nephrology 2007;12:130-4.

10. Wang AY, Woo J, Lam CW, et al. Associations of serum fetuin-A with malnutrition, inflammation, atherosclerosis and valvular calcification syndrome and outcome in peritoneal dialysis patients. Nephrol Dial Transplant 2005;20:1676-85.

11. Heimburger O, Stenvinkel P, Lindholm B. Nutritional effects and nutritional management of chronic peritoneal dialysis. In: Kopple JD, Massry SG. Nutritional management of renal disease. 2nded. Philadelphia: Lippincott Willians \& Wilkins; 2004. p. 477-511.

12. Martins C, Pecoits-Filho RFSP, Riella MC. Nutrição e diálise peritoneal. In: Martins C, Riella MC. Nutrição e o rim. Rio de Janeiro: Editora Guanabara Koogan; 2001. p. 132-48.

13. Baczynski R, Massry SG, Magott M, El-Belbessi S, Kohan $\mathrm{R}$, Brautbar N. Effect of parathyroid hormone on energy metabolism of skeletal muscle. Kidney Int 1985;28:722-7.

14. Garber AJ. Effects of parathyroid hormone on skeletal muscle protein and amino acid metabolism in the rat. J Clin Invest 1983;71:1806-21.

15. National Kidney Foundation. DOQI Peritoneal Dialysis Adequacy Works Group. NKF DOQI clinical practice guidelines for adequacy of peritoneal dialysis. Am J Kidney Dis 1997;30:S67-136.

16. National Kidney Foundation. K/DOQI Clinical Practice Guideline for Bone Metabolism and Disease in Chronic Kidney Disease. Am J Kidney Dis 2003;42:S1-S202.

17. Sociedade Brasileira de Cardiologia. IV Diretriz Brasileiras sobre Dislipidemia e Prevenção da Aterosclerose do Departamento de Aterosclerose da Sociedade Brasileira de Cardiologia. Arq Bras Cardiol 2007;88:2-19.

18. WHO, World Health Organization. Physical Status: The use and interpretation of antropometry. Report of a WHO Expert Committee. Geneva; 1995. 439 p.

19. Frisancho AR. New norms of upper limb fat and muscle areas for assessment of nutritional status. Am J Clin Nutr 1981;34:2540-5.

20. Kuczmarski MF, Kuczarisk RJ, Najjar M. Descriptive anthropometric reference data for older Americans. J Am Diet Assoc 2000;100:59-66.

21. National Kidney Foundation. K/DOQI Clinical practice guidelines for nutrition in chronic renal failure. Am J Kidney Dis 2000;35:S1-140.

22. Bressan J. Sistema de suporte à avaliação nutricional e prescrição de dietas. Agromídia Software - DietPro, 4th edition, CD-ROM; 2003.

23. Instituto Brasileiro de Geografia e Estatística. Tabela de composição de alimentos/IBGE. 4a ed. Rio de Janeiro: IBGE; 1996. 137 p.

24. Philippi ST. Tabela de composição de alimentos: suporte para decisão nutricional. Brasília: ANVISA; 2001. $133 \mathrm{p}$.

25. Grodstein GP, Blumenkratz MJ, Kopple JD. Glucose absorption during continuous ambulatory peritoneal dialysis. Kidney Int 1981;19:564-7.

26. Martins C. Insuficiência renal crônica. In: Lameu E. Clínica nutricional. Rio de Janeiro: Revinter; 2005. p. 869-88.

27. Dinamod S, Henrich W. Nutrition and peritoneal dialysis. In: Mitch WE, Klahr S. Nutrition and the kidney. Boston: Little Brown; 1988. p. 198-217.

28. Lacativa PGS, Filho PJMP, Gonçalves MDC, Farias MLF. Indicações de paratireoidectomia no 
hiperparatireoidismo secundário à insuficiência renal crônica. Arq Bras Endocrinol Metab 2003;47:644-53.

29. Araújo SM, Ambrosoni P, Lobao RR, et al. The renal osteodystrophy pattern in Brazil and Uruguay: an overview. Kidney Int Suppl 2003;63:S54-6.

30. Sherrard DJ, Hercz G, Pei Y, et al. The spectrum of bone disease in end-stage renal failure - an evolving disorder. Kidney Int 1993;43:436-42.

31. Delmez JA, Slatopolsky E, Martin KJ, Gearing BN, Harter HR. Minerals, vitamin D, and parathyroid hormone in continuous ambulatory peritoneal dialysis. Kidney Int 1982;21:862-7.

32. Billa V,ZhongA,Bargman J,VasS, WongPY, Oreopoulos DG. High prevalence of hyperparathyroidism among peritoneal dialysis patients: a review of 176 patients. Perit Dial Int 2000;20:315-21.

33. Malberti F, Marcelli D, Conte F, Limido A, Spotti D, Locatelli F. Parathyroidectomy in patients on renal replacement therapy: an epidemiological study. J Am Soc Nephrol 2001;12:1242-8.

34. Visser M, Deeg DJH, Lips P. Low vitamin D and high parathyroid hormone levels as determinants of loss of muscle strength and muscle mass (sarcopenia): the longitudinal aging study Amsterdam. J Clin Endocrinol Metab 2003;88:5766-72.
35. Ritz E, Boland, R, Kreusser W. Effects of vitamin D and parathyroid hormone on muscle: potential role in uremic myopathy. Am J Clin Nutr 1980;33:1522-9.

36. Rezende LTT, Cuppari L, Carvalho AB, et al. Nutritional status of hemodialysis patients with secondary hyperparathyroidism. Braz J Med Biol Res 2000;33:1305-11.

37. Prasad N, Gupta A, Sinha A, et al. Confounding effect of comorbidities and malnutrition on survival of peritoneal dialysis patients. J Ren Nutr 2010;20:384-91.

38. Lee CT, Tsai YC, Ng HY, et al. Association between $\mathrm{C}$-reactive protein and biomarkers of bone and mineral metabolism in chronic hemodialysis patients: a crosssectional study. J Ren Nutr 2009;19:220-7.

39. Stenvinkel P. Inflammation in end-stage renal failure: could it be trated? Nephrol Dial Transplant 2002;17:33-8.

40. Nordfors L, Heimbürger O, Lönnqvist F, et al. Fat tissue accumulation during peritoneal dialysis is associated with a polymorphism in uncoupling protein 2 . Kidney Int 2000;57:1713-9.

41. Santana APS, Lobão RRS, Draibe AS, Carvalho AB. Revisão: Osteodistrofia renal em pacientes submetidos à diálise peritoneal ambulatorial contínua. J Bras Nefrol 2002;24:97-102. 